\title{
Injury survey in Choi Kwang Do (CKD) martial art practitioners around the world: CKD is a safe form of training for adults
}

\author{
Yong-Seok Jee, Denny Eun* \\ Department of Physical Activity Design, Hanseo University, Seosan, Korea
}

Among the many sports and activities to choose from, martial arts are becoming increasingly popular for health and fitness. Due to the different nature of the various styles of martial arts, injuries are not uncommon. Though there have been studies on the injury rates of several martial art styles, there have been none regarding Choi Kwang Do (CKD), a noncompetitive martial art with relaxed and fluid movements designed to promote health and fitness for people of all ages. The purpose of this study was to examine the rate of injury for adults training in CKD to find out whether this is a safe style of martial art for adults. This study found the prevalence, causes, severity, and types of injuries from CKD practitioners around the world through an online survey targeting adults $(n=122)$, aged 18 or older, with varying years of training experi- ence. The annual rate of injury was 11.73 for every 100 CKD practitioners. There was no correlation between the length of training experience and injury. Training frequency and duration had no significant relationship with injury rates. A significant positive relationship between training intensity and injury existed $(P=0.009)$. The results of the study found that CKD can be an attractive option for adults of any age who are looking to learn a martial art or choose a physical activity with a low risk of injury, however the training intensity should be kept at a level that is not excessively high.

Keywords: Choi Kwang Do, Martial art, Adults, Health, Injury

\section{INTRODUCTION}

Certain sports and activities that were once enjoyed during a person's younger years often have to be relegated to the past as they age. When the physical demands of such sports or activities exceed an individual's capacity, there is a risk of injury. This is in addition to the constant risk of injuries due to the actual activities themselves, such as competitive sports and activities (Bledsoe et al., 2006; Gartland et al., 2005; Yang et al., 2012). Generally, older adults require a longer period of time to fully recover from injuries and may have a greater risk of injury than younger adults (Stevenson et al., 2000). Therefore, as a person becomes older, a greater importance is placed on safety, which may lead to avoiding certain types of physical activity. As a result, the kinds of activities that older adults can enjoy into their later years become more lim- ited. When also accounting for activities that include all three general categories of exercise which include aerobic training, resistance training, and flexibility training (Avers, 2016), the options may dwindle even further. Among the various sports and activities an individual can choose from, martial arts are increasingly becoming a more popular form of training for people of all ages (Ko and Yang, 2009). When training in martial arts, injuries can result from several reasons such as training habits and accidents, but people involved in competitions that involve full contact strikes are particularly more prone to injuries (Bledsoe et al., 2006; Covarrubias et al., 2015; Gartland et al., 2005; Zazryn et al., 2003). More specifically, there are also different rates of injuries among various styles of martial arts (McPherson and Pickett, 2010; Zetaruk et al., 2005). Generally, martial arts can be categorized as either 'hard' or 'soft.' The 'hard' martial arts, such as tae-
${ }^{*}$ Corresponding author: Denny Eun (iD https://orcid.org/0000-0002-9044-8655 Department of Physical Activity Design, Hanseo University, 46 Hanseo 1-ro, Haemi-myeon, Seosan 31962, Korea

Tel: +82-41-660-1545, Fax: +82-42-660-1088, E-mail: eun23415@gmail.com Received: December 19, 2017 / Accepted: January 17, 2018
This is an Open Access article distributed under the terms of the Creative Commons Attribution Non-Commercial License (http://creativecommons.org/licenses/by-nc/4.0/) which permits unrestricted non-commercial use, distribution, and reproduction in any medium, provided the original work is properly cited. 
kwondo (TKD), are based on using blocks and punches that can inflict significant damage to the bones or body parts of the opponent. The 'hard' martial arts tend to use fewer strikes and punches, but with more power. The 'soft' martial arts are based on redirecting the opponent's energy/attack and using more, but less powerful, punches and kicks. Tai chi chuan (TC), is an example of a 'soft' martial art (Brudnak et al., 2002; Burke et al., 2007). Most martial art styles fall into one or more categories: striking-based systems, grappling and throwing systems, weapons-based systems, and health-based systems (Terry, 2006).

Choi Kwang Do (CKD) is a modern martial art that relies more on flexibility and fluidity of movement as opposed to the more rigid motions of some traditional styles. CKD techniques employ natural bilateral movement and fluid sequential motion to develop maximum force on impact while minimizing pressure on the joints. The program is based on scientific principles and aims to promote optimum health, practical self-defense, and personal development for people of all capabilities, skills levels, and ages (http://choikwangdo.com/what_is_CKD.html) (Choi, 2011).

According to Terry (2006), CKD would be categorized as a striking-based art, as well as a health-based system. Although CKD would be categorized as a 'hard' martial art according to Brudnak et al. (2002) and Burke et al. (2007), its motions consist of fluid, circular movements and does not include full contact sparring or competitions. Studies have shown that $40.3 \%$ of martial art practitioners who were involved in mixed martial arts competitions incurred injuries (Bledsoe et al., 2006). Zetaruk et al. (2005) conducted a comparative study on five different martial art styles with the following results: Shotokan karate (29.8\%), Olympic style taekwondo (59.2\%), Aikido (51.1\%), kung fu (38.5\%), and TC (14.3\%). Among them, Aikido (27.7\%) had the highest incidence of major injuries followed by taekwondo (26.5\%), kung fu (17.9\%), karate (16.7\%), and TC (7.1\%). One of the reasons CKD was created was to reduce the risk of injuries by excluding competitive aspects, employing fluid techniques, and using the 10 components to promote health and fitness, self-defense, and personal development. However, no studies have been conducted to investigate the rate of injuries for CKD practitioners. The purpose of this study was to investigate the prevalence of injuries and to examine if this is a safe martial art for adults.

\section{MATERIALS AND METHODS}

\section{Subjects}

The participants for this study consisted of 122 current CKD
Table 1. Demographic data for the participants

\begin{tabular}{lccc}
\hline Variable & Male $(\mathrm{n}=82)$ & Female $(\mathrm{n}=40)$ & Total $(\mathrm{n}=122)$ \\
\hline Age $(\mathrm{yr})$ & $41.18 \pm 11.12$ & $39.05 \pm 13.18$ & $40.08 \pm 11.82$ \\
Height $(\mathrm{cm})$ & $175.78 \pm 7.44$ & $163.70 \pm 6.47$ & $171.82 \pm 9.11$ \\
Weight $(\mathrm{kg})$ & $81.84 \pm 12.62$ & $71.65 \pm 18.62$ & $78.50 \pm 15.54$ \\
\hline
\end{tabular}

Values are presented as mean \pm standard deviation.

practitioners (82 men, 40 women) aged 18 and older from 9 countries, which included Argentina, Australia, Colombia, Germany, India, Malaysia, South Korea, UK, and US. The demographic details are shown in Tables 1, 2.

\section{Survey}

Demographics, training volume, and injury details were obtained using the online survey consisting of 22 questions. Demographic information included age, sex, height, weight, country of residence, years of CKD training experience, current belt rank, and purpose for learning CKD. Questions regarding training volume included frequency, intensity, and duration. Lastly, injury details consisted of questions pertaining to the cause, diagnosis, number of injuries, severity, and area of injury.

E-mails were sent to CKD school owners in the countries previously mentioned which outlined the purpose of the study as well as the directions. The directions included asking their students to take an online survey with the inclusion criteria being 18 years of age or older and currently involved in CKD training. The school owners informed their students that participation in the study was voluntary and that their responses would be kept confidential, which were also stated in the survey. The participants were also informed of the purpose of the study and asked to sign an informed consent form approved by the Hanseo University College of Health Science Human Studies Committee. The investigatory online survey was conducted between February 4, 2016 to March 21,2016 . The link to the online survey was given only to those who expressed an intention to participate in the study.

\section{CKD training overview}

Safety is one of the most important aspects of CKD training. The techniques are designed to generate maximum power through the use of sequential motion while minimizing injuries to the joints. Competitions such as full contact sparring and tournaments are not a part of CKD training. A typical class is between 45 to $60 \mathrm{~min}$ and includes a combination of 10 components: warm up/cool down, stretching/flexibility, basic techniques, patterns, speed drills, defense drills, target training, close range de- 
Table 2. Characteristics of the participants

\begin{tabular}{|c|c|}
\hline Variable & No. $(\%)$ \\
\hline \multicolumn{2}{|l|}{ Nations } \\
\hline Argentina & $14(11.5)$ \\
\hline Australia & $14(11.5)$ \\
\hline Colombia & $1(0.8)$ \\
\hline Germany & $1(0.8)$ \\
\hline India & $1(0.8)$ \\
\hline Malaysia & $1(0.8)$ \\
\hline South Korea & $8(6.6)$ \\
\hline UK & $56(45.9)$ \\
\hline USA & $26(21.3)$ \\
\hline Total & $122(100)$ \\
\hline \multicolumn{2}{|l|}{ Belt rank } \\
\hline Color belts & $42(34.4)$ \\
\hline $1 \mathrm{st}$ & $24(19.7)$ \\
\hline 2nd & $16(13.1)$ \\
\hline $3 r d$ & $17(13.9)$ \\
\hline 4th & $12(9.8)$ \\
\hline 5th or above & $11(9.0)$ \\
\hline Total & $122(100)$ \\
\hline \multicolumn{2}{|l|}{ Experience duration (yr) } \\
\hline$<1$ & $12(9.8)$ \\
\hline $1-4$ & $47(38.5)$ \\
\hline $5-8$ & $18(14.8)$ \\
\hline $9-12$ & $18(14.8)$ \\
\hline $13-16$ & $11(9.0)$ \\
\hline $17-20$ & $7(5.7)$ \\
\hline$\geq 21$ & $9(7.4)$ \\
\hline Total & $122(100)$ \\
\hline \multicolumn{2}{|l|}{ Training purpose (multiple reply) } \\
\hline To become a professional instructor & $48(9.1)$ \\
\hline To enjoy as a hobby & $74(14)$ \\
\hline For social purposes & $39(7.4)$ \\
\hline To relieve stress & $67(12.7)$ \\
\hline To strengthen my mind & $57(10.8)$ \\
\hline To learn self defense & $93(17.6)$ \\
\hline For health and fitness & $107(20.2)$ \\
\hline To lose weight & $32(6.0)$ \\
\hline Others & $12(2.3)$ \\
\hline Total & $529(100)$ \\
\hline
\end{tabular}

fense, rehabilitation/strength training, and character development. The first component is warm-up/cool down exercises, which may include jumping jacks, running in place, lightly performing punches and kicks in the air, etc. This is followed by stretching and flexibility exercises which consist of 2 types: static and dynamic. Static stretching involves sustaining muscle-lengthening activities using body weight. Each position is held at the point of tension for 10-20 sec while the muscles are relaxed with deep abdominal breathing. Dynamic stretching consists of performing CKD movements slowly and using momentum to move joints throughout the entire range of motion to improve dynamic flexibility. The next component is basic techniques, which consist of basic punches, strikes, kicks, and blocks. The fourth component, patterns, involve preset combinations of basic techniques, which are different for each belt rank. The level of intensity in which the patterns are performed can be adjusted for various training purposes. Speed drills consist of a combination of offensive techniques that are intended to be performed at high speeds. Defense drills involve working with a partner to practice and develop skills needed for handling an attack or confrontation while wearing safety equipment. Target training uses equipment such as hand held targets and punching bags to apply CKD techniques against such objects of resistance. Close range defense is practiced in the event a student is grabbed by an attacker. Rehabilitation and strength training involves returning the body's function to its preinjury state and increasing muscle strength and endurance. Lastly, character development trains the mind to adopt a positive attitude and belief system.

For cardiovascular development, basic techniques, patterns, speed drills, and defense drills are often performed for a certain period of time at a level of intensity to bring the heart rate up to $60 \%-80 \%$ of the maximum heart rate for $20 \mathrm{~min}$. For anaerobic development, patterns, speed drills, and target training are performed at maximum speed for 1 or $2 \mathrm{~min}$ and then given a period of rest that is twice the length of time spent in activity. Conditioning, or strength training, involves additional target training exercises, calisthenics, and plyometric activities.

\section{Statistical analyses}

Statistical analysis was conducted with the IBM SPSS ver. 18.0 (IBM Co., Armonk, NY, USA). All data are reported as mean \pm standard deviation and as mean change over baseline. Prior to the main analysis, the Kolmogorov-Smirnov test was used to determine the normality of distribution of the examined variables. Since the data was not normally distributed, nonparametric Mann-Whitney $U$-test was used for comparing the variables. The examined variables included participant training experience, training volume, number of injuries, severity of injuries, injured body parts, causes of injuries, and diagnoses of injuries. Frequency analysis was used as an analysis method and verification was performed at a significance level of $P<0.05$. 


\section{RESULTS}

\section{Demographic data of the participants}

As shown in Table 1, the mean age, height, and weight were $40.08 \pm 11.82$ years, $171.82 \pm 9.11 \mathrm{~cm}$, and $78.50 \pm 15.54 \mathrm{~kg}$, respectively. Of the 122 participants, there were 82 men and 40 women.

\section{Characteristics of the participants}

Table 2 describes the characteristics of the participants. Among the 9 countries, the highest number of participants was from the UK (45.9\%). Color belts accounted for more than any other rank (34.4\%), followed by 1st degree black belts (19.7\%). There was a wide range of training experience, but most participants had $1-4$ years $(38.5 \%)$. The average training experience of all the participants was 7.96 years and the most common reason for training was for health and fitness (20.2\%), followed by self-defense $(17.6 \%)$.

\section{CKD training volume}

Although the belt curriculum is uniform across all CKD schools, each school is led by different instructors who may have different ways of teaching class. To gather data on the training volume for each participant, questions regarding the frequency, intensity, and

Table 3. Training volume of Choi Kwang Do martial art

\begin{tabular}{lc}
\hline Variable & No. (\%) \\
\hline Frequency & $11(9.0)$ \\
Once a week or less & $46(37.7)$ \\
Twice a week & $35(28.7)$ \\
Three times a week & $30(24.6)$ \\
Four times a week or more & $122(100)$ \\
Total & \\
Intensity & $0(0)$ \\
Extremely low & $0(0)$ \\
Very low & $2(1.6)$ \\
Low & $58(47.5)$ \\
Moderate & $44(36.1)$ \\
High & $15(12.3)$ \\
Very high & $3(2.5)$ \\
Extremely high & $122(100)$ \\
Total & \\
Duration (min) & $4(3.3)$ \\
$<45$ & $83(68.0)$ \\
$45-60$ & $35(28.7)$ \\
$>60$ & $122(100)$ \\
Total &
\end{tabular}

duration were asked in the survey as shown in Table 3. The most common CKD training volume was a frequency of twice a week (37.7\%) with moderate intensity (47.5\%), and a duration of $45-$ $60 \mathrm{~min}(68.0 \%)$. To obtain specific figures, values were given for 7 levels of intensity: extremely low (1), very low (2), low (3), moderate (4), high (5), very high (6), extremely high (7). For duration, 3 values were used to obtain a specific number of minutes: $45 \mathrm{~min}$ for ' $45 \mathrm{~min}$ or less,' $52.5 \mathrm{~min}$ for ' 45 to $60 \mathrm{~min}$,' and $90 \mathrm{~min}$ for 'more than 60 min.' Using these values, the participants in this study trained with a frequency of 2.7 times per week, with moderate to high intensity, for a duration of $63.4 \mathrm{~min}$ per training session. Per week, this amounts to $2.85 \mathrm{hr}$ of CKD training.

There was no significant correlation between training frequency and duration on injury. However, there was a significant correlation $(P=0.009)$ between training intensity and injury as shown in Table 4.

\section{Physical injury rates, severity, and body parts from CKD training}

To provide a more standardized way of expressing the injury rate of CKD practitioners, the annual number of injuries per 100 people was calculated with a formula used in a study by Birrer and Halbrook (1988) which investigated the injury rate of martial arts practitioners from a 5 -year national survey. The annual rate of injury per $100 \mathrm{CKD}$ practitioners was $11.73\left(\frac{114 \text { (total \# of injuries) }}{122 \text { (total \# of participants) }}\right.$

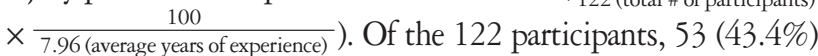
sustained a total of 114 injuries during their entire training experience in $\mathrm{CKD}$. The participants in this study had an average training frequency of 2.7 times per week and a duration of 63.4 min per session (171.18 min per week). $43.4 \%$ of those who participated in the study experienced a physical injury once (39.69\%). Participants who experienced injury chose from among 6 degrees of severity ranging from 'very minor' (11.32\%), 'minor' (35.88\%), 'somewhat minor' (26.49\%), 'somewhat severe' (16.96\%), 'severe' (3.70\%), and 'very severe' (5.65\%), all of which were self-reported. The body parts that were most involved in injuries were 'multi

Table 4. Comparative results of frequency, intensity, and duration from Choi Kwang Do training volume

\begin{tabular}{lccc}
\hline Group & Frequency & Intensity & Duration (yr) \\
\hline Not injured $(\mathrm{n}=69)$ & $2.69 \pm 0.97$ & $4.50 \pm 0.72$ & $2.27 \pm 0.54$ \\
Injured $(\mathrm{n}=53)$ & $2.69 \pm 0.92$ & $4.88 \pm 0.88$ & $2.23 \pm 0.47$ \\
$Z$ & -0.033 & -2.621 & -0.525 \\
$P$-value & 0.974 & 0.009 & 0.599
\end{tabular}

Values are presented as mean \pm standard deviation. Results were analyzed by Mann-Whitney U-test. 
parts' (47.19\%) followed by 'lower limb' (18.86\%), 'ankle, foot, or toe', 'hand, wrist, or finger', and 'no reply' which were equally $7.55 \%$ each, and 'back' (1.6\%), and 'head,' 'upper limb,' 'hip or groin,' and 'others' which equally accounted for $1.89 \%$ each. Further details of injuries are shown in Table 5.

\section{Causes of injury and diagnosis from CKD training}

Improper technique was cited as the most common cause of injury $(27.7 \%)$. The most common diagnoses of injuries were muscle sprains (21.9\%) followed by bruises (14.3\%) and ligament sprains (12.4\%). A complete list of the causes and diagnosis of injuries is shown in Table 6 .

Table 5. Physical injury rates and severity from Choi Kwang Do training

\begin{tabular}{lc}
\hline Variable & No. (\%) \\
\hline Physical injury & \\
No & $69(56.6)$ \\
Yes & $53(43.4)$ \\
Total & $122(100)$ \\
Injury frequency & \\
No reply & $1(1.9)$ \\
Once & $21(39.7)$ \\
Twice & $15(28.4)$ \\
Three times & $11(20.8)$ \\
Four times & $2(3.8)$ \\
Others & $3(5.7)$ \\
Total & $53(100)$ \\
Injury everity & \\
Very minor & $6(11.3)$ \\
Minor & $19(35.8)$ \\
Somewhat minor & $14(26.4)$ \\
Somewhat severe & $9(17.0)$ \\
Severe & $2(3.8)$ \\
Very severe & $3(5.7)$ \\
Total & $53(100)$ \\
Injured body part & \\
No reply & $53(100)$ \\
Head & \\
Upper limb & $4(7.5)$ \\
Hand, wrist, or finger & $1(1.9)$ \\
Back & $1(1.9)$ \\
Hip or groin & $4(7.5)$ \\
Lower limb & $2(3.8)$ \\
Ankle, foot, or toe & $1(1.9)$ \\
Multiparts & $10(18.9)$ \\
Others & $4(7.5)$ \\
Total & $1(1.9)$ \\
\hline & \\
\hline
\end{tabular}

\section{DISCUSSION}

The most common reason the participants of this study chose to train in CKD was for health and fitness. This can be attributed to the growing trend of martial arts becoming an increasingly popular recreational activity for all ages (Ko and Yang, 2009). Each martial art style has its own risks and benefits, but since no study to date has been conducted on CKD, this study specifically focused on the risk of injuries of adult CKD practitioners aged 18 years and above. Among the 122 participants currently training in $\mathrm{CKD}, 43.4 \%$ reported having incurred at least one injury since beginning their training. However, the vast majority of injuries (73.69\%) were categorized as more or less minor in severity and this includes those who have trained in CKD for over 21 years. This may be due to the fact that CKD does not engage in any kind of sparring, competitions, or tournaments, as most documented injuries from martial arts occur during such activities (Birrer and Halbrook, 1988).

When examining the rate of injury in relation to the amount of CKD training experience participants had, those with 13-16 years of experience had the highest incidence of injury (63.6\%). Most studies have shown that the amount of experience or expo-

Table 6. Injury causes and diagnoses of the injured persons $(n=53)$ (multiple reply)

\begin{tabular}{lc}
\hline Variable & No. of cases (\%) \\
\hline Cause of Injury & \\
Inadequate warm-up & $8(9.6)$ \\
Over-training & $14(16.9)$ \\
Improper technique & $23(27.7)$ \\
Overexertion & $15(18.1)$ \\
Inadequate practice & $5(6.0)$ \\
Others & $18(21.7)$ \\
Total & $83(100)$ \\
Diagnosed Injury & \\
Bone fracture & $9(8.6)$ \\
Ligament sprain & $13(12.4)$ \\
Muscle strain & $23(21.9)$ \\
Tendon strain & $11(10.5)$ \\
Abrasion & $3(2.9)$ \\
Bruise & $15(14.3)$ \\
Muscle cramp & $3(2.9)$ \\
Bleeding & $4(3.8)$ \\
Inflammation & $11(10.5)$ \\
Rupture & $3(2.9)$ \\
Others & $10(9.5)$ \\
Total & $105(100)$ \\
\hline &
\end{tabular}


Table 7. Injuries categorized by Choi Kwang Do training experience

\begin{tabular}{lcrcccc}
\hline $\begin{array}{c}\text { Experience } \\
\text { duration (yr) }\end{array}$ & $\begin{array}{c}\text { Not } \\
\text { injured }\end{array}$ & Injured & Total & $\begin{array}{c}\text { Not } \\
\text { injured rate (\%) }\end{array}$ & $\begin{array}{c}\text { Injured } \\
\text { rate (\%) }\end{array}$ & $\begin{array}{c}\text { Total } \\
\text { rate (\%) }\end{array}$ \\
\hline$<1$ & 11 & 1 & 12 & 91.7 & 8.3 & 100 \\
$1-4$ & 24 & 23 & 47 & 51.1 & 48.9 & 100 \\
$5-8$ & 12 & 6 & 18 & 66.7 & 33.3 & 100 \\
$9-12$ & 9 & 9 & 18 & 50.0 & 50.0 & 100 \\
$13-16$ & 4 & 7 & 11 & 36.4 & 63.6 & 100 \\
$17-20$ & 4 & 3 & 7 & 57.1 & 42.9 & 100 \\
$\geq 21$ & 5 & 4 & 9 & 55.6 & 44.4 & 100 \\
\hline
\end{tabular}

sure to any activity is directly related to injuries since there are more chances for incurring injuries. However, these results show that a clear positive relationship between experience and injury did not exist as shown below in Table 7 .

When observing training volume of the participants, practicing two to three times a week for $45-60$ min per class with a training intensity between moderate and high were most common. Such results may be explained due to the fact that CKD training is meant to be progressive in that practitioners should always be challenged, but not to the extent that it overtaxes the body. Since overexertion can cause damage, CKD classes aim for a moderate to high level of intensity. In order to include the necessary components for aerobic development, anaerobic development, flexibility training, and conditioning, $45 \mathrm{~min}$ or more is usually required. Learning new techniques, patterns, or other parts of the CKD curriculum for each belt rank also requires additional time.

The body parts that were most involved in injuries consisted of more than just one part, including the lower limbs, ankles, feet, toes, hands, wrists, and fingers. Much of these results were in line with other studies that investigated the common areas of injury from martial arts practice (Burks and Satterfield, 1998; Buschbacher and Shay, 1999; Terry, 2006).

As with any martial art or physical activity, proper form and movement are essential to preventing injury and according to the participants, the most commonly cited causes of injury were improper technique, overexertion, and other reasons such as board breaking, accidents from defense drills, and problems with safety equipment. Due to the relatively high number of injuries from board breaking, CKD International Headquarters decided to exclude all forms of board breaking. Among the various diagnoses of injuries, muscle strains were the most common. A possible explanation for this may be due to the high percentage of participants who indicated that improper technique and overexertion were the causes of their injuries.

It is important to note that differing definitions and calcula- tions of injuries may make comparisons difficult. To put this in perspective, the incidence of injury can be expressed in terms of the number of injuries within a set period of time. For example, a study that investigated the number of injuries during Muay Thai kickboxing competitions showed that there was an average of one injury for every 42 min while another study calculated the injury rate of a karate-based system at 2.70 injuries per 1,000 hr of practice (Buschbacher and Shay, 1999; Gartland et al., 2005). Other studies have expressed injury rates as the number of injuries per 100 people per year (Birrer and Halbrook, 1988; Destombe et al., 2006). This study calculated the annual rate of injury per 100 practitioners.

A study by Tenvergert et al. (1992) looked at the injury rates of volleyball, soccer, gymnastics, martial arts over a period of 7 years and found that the annual rate of injury was highest in soccer (59.2/100/yr) followed by volleyball (16.7/100/yr), gymnastics (14.9/100/yr), and martial arts (9.1/100/yr) (Destombe et al., 2006; Tenvergert et al., 1992). Another study by Destombe et al. (2006) surveyed 186 karate athletes and found an annual injury rate of 44.6/100. The results of this study showed that the injury rate for adult CKD martial art practitioners was $11.73 / 100 / \mathrm{yr}$, which indicates that it is a relatively safe form of physical activity.

Though it may seem obvious that the low rate of injury from CKD training is due to the lack of sparring, a study by Koh (2014) showed that $73 \%$ of taekwondo athletes who trained in sport-poomsae, which involves individually performing a set pattern of techniques against an imaginary opponent, incurred chronic overuse injuries (COI) with an average training volume of 3.2 days per week and $71 \mathrm{~min}$ per day. COI results when the rate of healing cannot keep up with the damage incurred from repetitive stress to the bones and musculotendinous tissues (Shuer and Dietrich, 1997; Yang et al., 2012). Such injuries were not caused by sparring competitions, but instead were caused from repeatedly performing certain techniques, especially high kicks that involve rapid snapping at the knee joints (Koh, 2014). Considering the similarities in terms of training without sparring, the low rate of injury from CKD training may be attributed to the fluid movements and lack of lockout techniques. Several other studies have also shown that TC, another martial art style that does not engage in sparring, has a relatively low rate of injuries when compared to other competitive martial arts (McPherson and Pickett, 2010; Terry, 2006; Zetaruk et al., 2005).

According to a study by Zetaruk et al. (2005), adults trained in various martial art styles for an average of $5.4 \mathrm{hr}$ per week with a tendency for increased injury in relation to increased training 
time. However, the results of this study showed that training frequency and duration did not significantly affect the rate of injury, which is also consistent with a study by Koh (2014) described earlier. The scoring criterion for poomsae focuses on aspects such as balance, strength, speed, rhythm, and expression of energy. The only training characteristic that showed to have a significant relationship with injury rates in CKD was training intensity $(P=$ 0.009). This suggests that the rate of injury may be lowered by keeping the level of training intensity from being too high. Overall, the low annual injury rate of $11.73 / 100$ suggests that CKD is a safe martial art that adults of all ages can participate in.

Although there are conflicting results regarding age and injury from martial arts training, studies generally seem to show that injuries increase as age increases (Bledsoe et al., 2006; Destombe et al., 2006; Zetaruk et al., 2005). The current study only investigated the incidence of injuries for adult CKD practitioners aged 18 years and above so it may be worth conducting a future study for those younger than 18 years. Considering the fact that the years of $\mathrm{CKD}$ training that participants had ranged from less than 1 year to over 21 years, minor injuries may have been forgotten. Instead of asking the participants for the number of injuries incurred throughout their entire CKD training experience, it may have been more useful to obtain information regarding injuries experienced during the past year. That would have also allowed for an analysis of the relationship between training experience and rate of injury. In addition, the cause, diagnosis, and severity of injury were all self-reported. Since each individual may have different definitions of what is minor or severe, as well as not knowing how to properly diagnose or understand the cause of injury, future studies may benefit by setting clear distinctions on the levels of injury severity and obtaining a doctor's diagnosis.

\section{CONFLICT OF INTEREST}

No potential conflict of interest relevant to this article was reported.

\section{ACKNOWLEDGMENTS}

This study was supported by the Research Grant of Hanseo University in 2016. We also convey our appreciation to those who participated in this study around the world.

\section{REFERENCES}

Avers D. Aerobic exercise for older adults. Annu Rev Gerontol Geriatr 2016;36;123-154.

Birrer RB, Halbrook SP. Martial arts injuries. The results of a five year national survey. Am J Sports Med 1988;16:408-410.

Bledsoe GH, Hsu EB, Grabowski JG, Brill JD, Li G. Incidence of injury in professional mixed martial arts competitions. J Sports Sci Med 2006; 5(CSSI):136-142.

Brudnak MA, Dundero D, Van Hecke FM. Are the 'hard' martial arts, such as the Korean martial art, TaeKwon-Do, of benefit to senior citizens? Med Hypotheses 2002;59:485-491.

Burke DT, Al-Adawi S, Lee YT, Audette J. Martial arts as sport and therapy. J Sports Med Phys Fitness 2007;47:96-102.

Burks JB, Satterfield K. Foot and ankle injuries among martial artists. Results of a survey. J Am Podiatr Med Assoc 1998;88:268-278.

Buschbacher RM, Shay T. Martial arts. Phys Med Rehabil Clin N Am 1999;10:35-47.

Choi Kwang Do. What is Choi Kwang Do? [Internet]. Kennesaw (GA): Choi Kwang Do Martial Art International; 2011 [cited 2017 Jun 11]. Available from: http://choikwangdo.com/what_is_CKD.html.

Covarrubias N, Bhatia S, Campos LF, Nguyen DV, Chang EY. The relationship between Taekwondo training habits and injury: a survey of a collegiate Taekwondo population. Open Access J Sports Med 2015;6: 121-127.

Destombe C, Lejeune L, Guillodo Y, Roudaut A, Jousse S, Devauchelle V, Saraux A. Incidence and nature of karate injuries. Joint Bone Spine 2006;73:182-188.

Gartland S, Malik MH, Lovell M. A prospective study of injuries sustained during competitive Muay Thai kickboxing. Clin J Sport Med 2005;15:34-36.

Ko YJ, Yang JB. The globalization of martial arts: the change of rules for new markets. Rev Artes Marciales Asiáticas 2009;4:8-19.

Koh JO. Prevalence of chronic overuse injuries in sport-poomsae Taekwondo competitors in North America. J Korean Phys Educ Assoc Girl Women 2014;28:19-33.

McPherson M, Pickett W. Characteristics of martial art injuries in a defined Canadian population: a descriptive epidemiological study. BMC Public Health 2010;10:795.

Shuer ML, Dietrich MS. Psychological effects of chronic injury in elite athletes. West J Med 1997;166:104-109.

Stevenson MR, Hamer P, Finch CF, Elliot B, Kresnow M. Sport, age, and sex specific incidence of sports injuries in Western Australia. Br J Sports Med 2000;34:188-194.

Tenvergert EM, Ten Duis HJ, Klasen HJ. Trends in sports injuries, 1982- 
1988: an in-depth study on four types of sport. J Sports Med Phys Fitness 1992;32:214-220.

Terry CM. The martial arts. Phys Med Rehabil Clin N Am 2006;17:645676.

Yang J, Tibbetts AS, Covassin T, Cheng G, Nayar S, Heiden E. Epidemiology of overuse and acute injuries among competitive collegiate ath- letes. J Athl Train 2012;47:198-204.

Zazryn TR, Finch CF, McCrory P. A 16 year study of injuries to professional boxers in the state of Victoria, Australia. Br J Sports Med 2003; 37:321-324

Zetaruk MN, Violán MA, Zurakowski D, Micheli LJ. Injuries in martial arts: a comparison of five styles. Br J Sports Med 2005;39:29-33. 